\title{
Thermal Stability and Crystallization Behavior of Contaminated Recycled Polypropylene for Food Contact
}

Isabelly Veroneze

Federal University of Sao Carlos: Universidade Federal de Sao Carlos

Akemi Letícia

Federal University of Sao Carlos: Universidade Federal de Sao Carlos

Sandra Andrea Cruz ( $\nabla$ sandra.cruz@ufscar.br)

Universidade Federal de Sao Carlos Centro de Ciencias Exatas e de Tecnologia https://orcid.org/00000002-5548-0166

\section{Research Article}

Keywords: Polypropylene (PP), Thermal Stability, Crystallization, Food Contact, thermogravimetric (TGA)

Posted Date: December 8th, 2021

DOI: https://doi.org/10.21203/rs.3.rs-1108252/v1

License: (a) (1) This work is licensed under a Creative Commons Attribution 4.0 International License. Read Full License

Version of Record: A version of this preprint was published at Journal of Polymers and the Environment on April 21st, 2022. See the published version at https://doi.org/10.1007/s10924-022-02447-9. 
Isabelly Bertochi Veroneze ${ }^{a}$, Letícia Akemi Onoue ${ }^{\mathrm{a}}$ and Sandra Andrea Cruz ${ }^{\mathrm{a}^{*}}$,

5

6

a. Chemistry Department, Center for Exact Sciences and Technology, Federal University

7 of São Carlos, Rodovia Washington Luís, Km 235, 10 SP-310, São Carlos, Brazil.

8

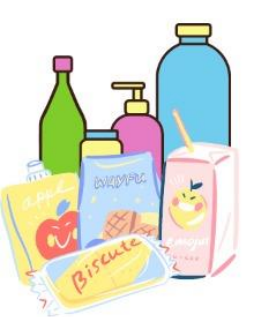

PP packaging

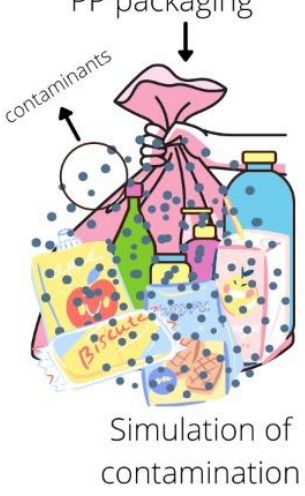

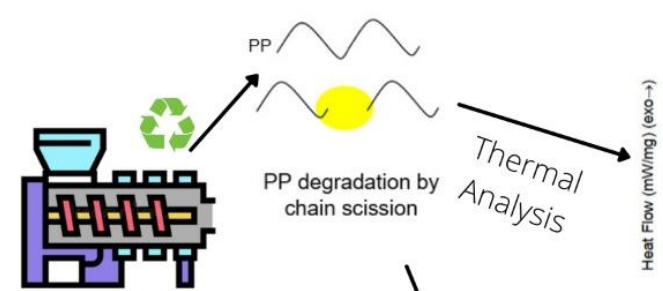

Reprocessing

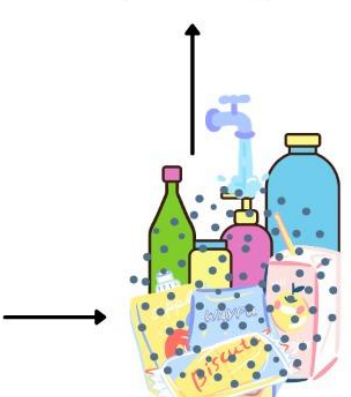

Decontamination by whashing

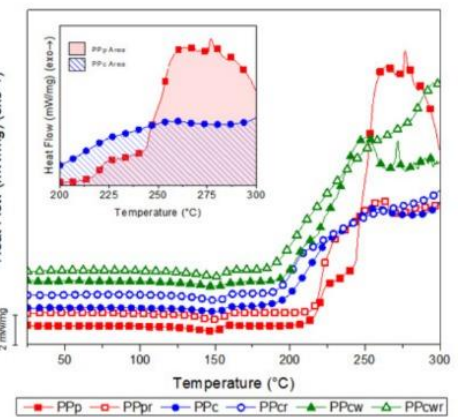

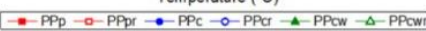

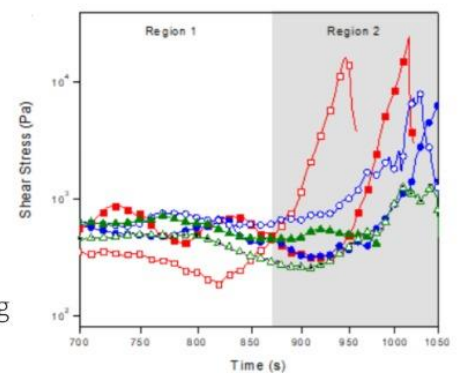


Polypropylene is one of the most widely used polymers, especially in the food packaging industry, which causes negative environmental effects. Recycling is a good option to partially solve this environmental problem. Thus, the polymer was contaminated with a cocktail to simulate the conditions of disposal and recycling following FDA guidelines.

The influence of contaminants on recycled PP was analyzed by quiescent and nonquiescent crystallization. It was found that the contaminants alter the crystallization

flow since longer induction times were observed for all contaminated samples. Also, the thermal behavior was performed considering that the thermogravimetric (TGA) results indicated an increase in the stability with the presence of contaminants. Therefore, a deep investigation using the induced oxidation time (OIT) and induced oxidation temperature (OITD) was performed. The contaminants play an important role in the 


\section{INTRODUCTION}

Polypropylene (PP) is one of the most consumed polymers in several industrial segments, from toys to automotive parts, due to its good properties and relatively low cost[1]. Unfortunately, its vast application as fast disposal packaging has increased the volume of municipal solid waste, contributing to the aggravation of environmental problems. Additionally, with the COVID-19 pandemic scenario, e-commerce, food deliveries and personal protective equipment have significantly increased, according to Parashar and Hait [2]. Safety concerns about food and personal contamination led to a preference of consumers in plastic containers and bags[3]. Thus, in the current and postpandemic scenario, a correct destination for waste is essential, and in this sense, recycling is part of the solution by closing the cycle and returning to its original application.

However, the use of recycled plastics for direct food contact is restricted by agencies such as the Food and Drug Administration (FDA) and the National Health Surveillance Agency (ANVISA), in Brazil and MERCOSUL. The concern was related to the migration of contaminants from post-consumer use to food contact, putting the well-being of consumers at risk[4,5]. An additional aspect when using recycled materials in direct contact with food is the presence of degradation products, defined as non-intentionally added substances (NIAS) that can migrate into food. The high shear rates and temperatures employed in the recycling process may increase the degradation process, which leads to a change in the physical-chemical negatively affected properties of the polymers restricting their applicability[6-13]. Its complexity comes from the fact that the formed compounds may be the degradation result of (i) the polymer chain, (ii) the intentionally added substances (IAS), (iii) NIAS and (iv) the reaction between all of them. 
A recent work published by Paiva et al. [6] analyzed the presence of NIAS in the same samples studied in this work. Polypropylene samples were subjected to forced contamination and a recycling process, which led to the identification of 45 different volatile compounds. Recently, a study[6] on the extractability/migration of contaminants from polypropylene (PP) samples into food simulants and the influence of these contaminants on the molecular structure of recycled PP was developed. The authors concluded that the high temperatures and shear rates used in the recycling process, when in contact with the residual contaminants, alter the molecular structure of this polymer.

This concern is more critical for PP due to the higher contaminant sorption capacity and its low thermal resistance when compared to poly(ethylene terephthalate) $[9,14]$. Therefore, these are additional factors in the recycling of contaminated polypropylene to be considered for direct contact with food. As described by Palkopoulu et al.[15], the polyolefins have reduced thermal stability, and this may contribute to an increase in the degradation process. Although recycling is environmentally friendly, it is possible to produce materials whose properties are negatively affected by this process, restricting their applicability[15].

The decrease in molar mass and deterioration of mechanical properties as a result of degradation becomes an economic disadvantage for the application of recycled PP when compared to pristine material [16-22]. Therefore, understanding the effect of contaminants on the thermal properties of polypropylene is essential to develop a way to add more value to recycled material that makes it suitable for reintroduction into the packaging market [17]. 
Therefore, this study aims to evaluate the degradation of polypropylene in the presence of contaminants in different stages of the recycling process, such as washing and reprocessing. The effect of the presence of contaminants on PP was analyzed by quiescent and nonquiescent crystallization, as well as by thermal analysis.

\section{METHODOLOGY}

The methodology of this work was developed into three main steps as follows: Step 1 consists of contaminating the PP with a cocktail of surrogates and recycling it; In Step 2, the efficiency of the recycling process was evaluated by an extractability test using different food simulants; In Step 3, given the presence of contaminants, the molecular structure was evaluated by rheometry after the recycling process.

\subsection{Materials}

In this work, polypropylene was used in pellet form (Prism 2400) supplied by Braskem S.A, Brazil. It presents a melt flow index (MFI) of $20 \mathrm{~g} / 10 \mathrm{~min}$ (ASTM 1238, $230^{\circ} \mathrm{C}, 2.16 \mathrm{~kg}$ ) and a density of $0.902 \mathrm{~g} / \mathrm{cm}^{3}$ (ASTM D 792). The chemicals used for contamination were chloroform (Vetec, 99.8\%, CAS No. 67663), toluene (Vetec, 99.5\%, CAS No. 108883), benzophenone (Acros Organics, 99\%, CAS No. 119619), tetracosane (Merck, 99\%, CAS No. 646311) and heptane (Synth, 99\%, CAS No. 1422825). Ethanol (Merck, 99\%) and acetic acid (Merck, 99\%) were employed to prepare the food simulants, with $10 \%(\mathrm{v} / \mathrm{v})$ ethanol and $3 \%(\mathrm{w} / \mathrm{v})$ acetic acid respectively in MiliQ water. 


\subsection{Methods}

\subsubsection{Contamination and Recycling Process}

112 To study the influence of the recycling process, the samples were subjected to forced 113 contamination according to Food and Drugs Administration (FDA) regulations [4]. 114 Therefore, the PP pellets were exposed to a contaminant cocktail and placed in a 115 hermetically sealed system kept in constant stirring at $40^{\circ} \mathrm{C}$ for 14 days to ensure uniform sample contamination (PPc). The contaminant cocktail composition is shown in

117 Table 1.

118 After contamination, contaminated pellets went through the process of washing with water for 10 minutes, followed by a second wash with sodium hydroxide for 5 minutes and, finally, again with water for 10 minutes. This procedure was based on the work of Garcia et al. [9]. 
Table 1: Contaminants, properties and concentration of the contaminants used in the cocktail.

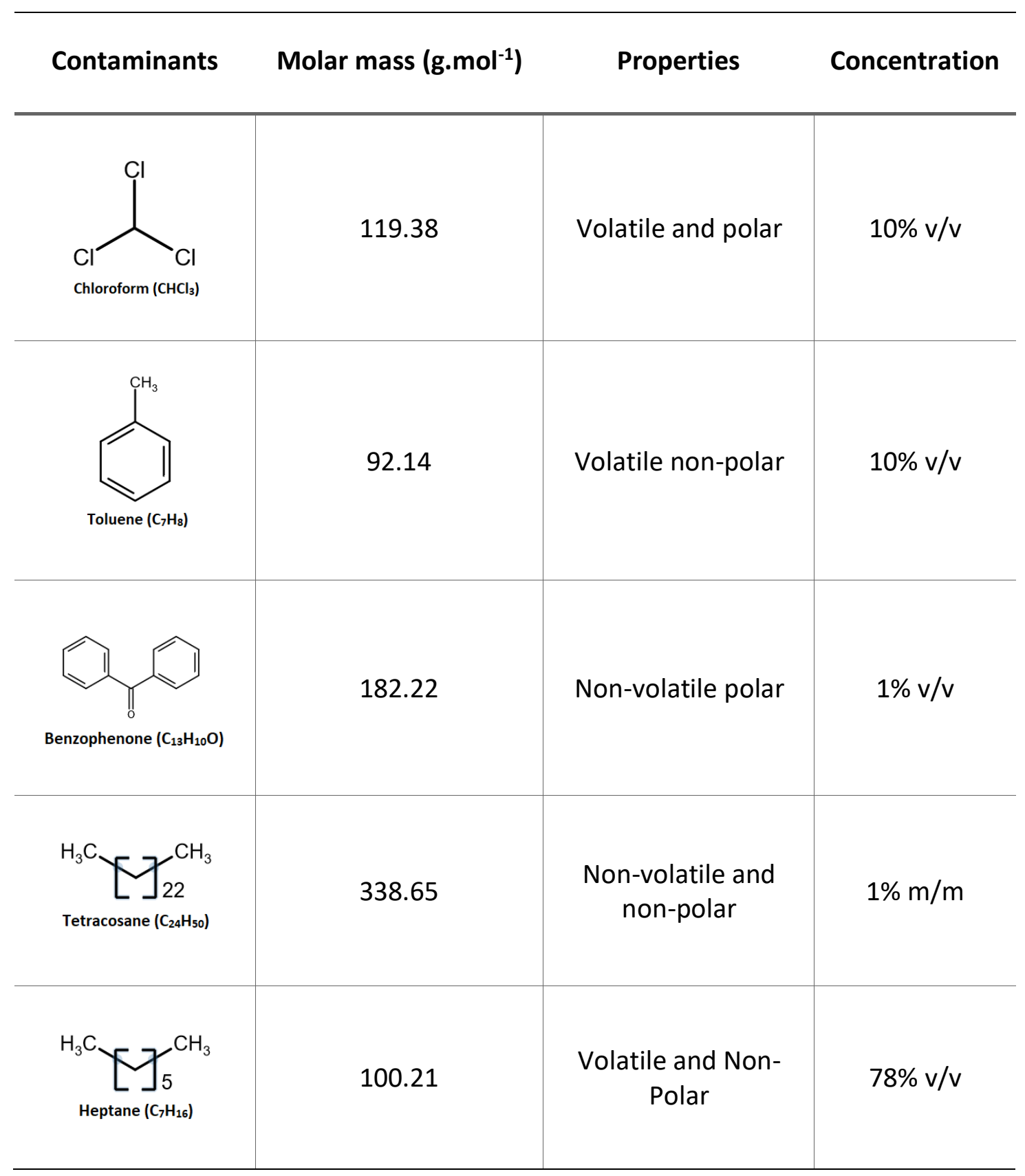

Subsequently, the contaminated material was reprocessed in a single-screw extruder (AX Plastics) $(D=16 \mathrm{~mm}, L / D=26)$. The screw speed used was $30 \mathrm{rpm}$ and the temperatures used were 180,190 , and $210^{\circ} \mathrm{C}$. The different processes led to a set of six samples presented in Table 2. 
Table 2: Nomenclature of the PP samples used in this work.

\begin{tabular}{c|c}
\hline Samples & Nomenclature \\
\hline Pristine PP & PPp \\
\hline Pristine -reprocessed PP & PPpr \\
\hline Contaminated PP & PPc \\
\hline Contaminated and reprocessed PP & PPcr \\
\hline Contaminated and washed PP & PPcw \\
\hline Contaminated, washed and reprocessed PP & PPcwr \\
\hline
\end{tabular}

\subsubsection{Thermal analysis}

Differential scanning calorimetry (DSC)

The analysis was performed in a DSC 203 F3-Maia (Netzsch). The samples were subjected to a three-step sweep: heating from $20^{\circ} \mathrm{C}$ to $180^{\circ} \mathrm{C}$, with a five-minute isotherm at $180^{\circ} \mathrm{C}$, followed by cooling from $180^{\circ} \mathrm{C}$ to $20^{\circ} \mathrm{C}$, with a two-minute isotherm at $20^{\circ} \mathrm{C}$ followed by a second heating using the same parameters as the first step. The analyses were carried out at a heating rate of $10^{\circ} \mathrm{C} \cdot \mathrm{min}^{-1}$ and an inert gas atmosphere $\left(\mathrm{N}_{2}\right)$ with a flow of $50 \mathrm{~mL} \cdot \mathrm{min}^{-1}$. The crystallinity degree data was calculated as follows:

$$
X_{C}=\frac{\Delta H_{m}}{\Delta H_{0}} \times 100 \%
$$

where $X_{C}$ is the crystallinity degree, $\Delta H m$ is the melting enthalpy obtained by the integration of the surface of the DSC curve related to the melting process and $\Delta \mathrm{H}_{0}$ expresses the heat fusion of perfectly crystallized PP (209 KJ/g)[23]. 
Oxidation Induction Time (OIT)

Analyses were performed in a DSC 203 F3-Maia (NETZSCH). The parameters for the conventional OIT technique were as follows: heating from $20^{\circ} \mathrm{C}$ to $200^{\circ} \mathrm{C}$ under an inert gas atmosphere $\left(\mathrm{N}_{2}\right)$ with a heating rate of $10^{\circ} \mathrm{C} \cdot \mathrm{min}^{-1}$, followed by a five-minute isotherm where it was carried out the exchange of gases, from $\mathrm{N}_{2}$ to $\mathrm{O}_{2}$. After the exchange, the samples remained in an isotherm for 35 minutes in an oxidizing atmosphere at $200^{\circ} \mathrm{C}$, the gas flow used was $50 \mathrm{~mL} \cdot \mathrm{min}^{-1}$.

\section{Oxidation Induction Time - Dynamic Regime (OITD)}

OITD analyses were performed in a DSC 203 F3-Maia (NETZSCH). The analysis conditions used were the following: a single heating from $20^{\circ} \mathrm{C}$ to $300^{\circ} \mathrm{C}$ under an oxidizing gas atmosphere $\left(\mathrm{O}_{2}\right)$ with $50 \mathrm{~mL} \cdot \mathrm{min}^{-1}$ gas flow and $10^{\circ} \mathrm{C} \cdot \mathrm{min}^{-1}$ heating rate.

\section{Thermogravimetric analysis (TGA)}

Thermogravimetric analyzes were performed on a TG 209 F3-Tarsus (NETZSCH). The samples were subjected to heating from $40^{\circ} \mathrm{C}$ to $600^{\circ} \mathrm{C}$ under an oxidative atmosphere $\left(\mathrm{O}_{2}\right)$ with a gas flow of $40 \mathrm{~mL} \cdot \mathrm{min}^{-1}$ and a heating rate of $10^{\circ} \mathrm{C} \cdot \mathrm{min}^{-1}$.

\section{Rheological analysis}

For crystallization analysis under nonquiescent conditions, a parallel plate rheometer (Anton Paar MCR 305) was used. The initial temperature used in the test was $190^{\circ} \mathrm{C}$ with $1 \%$ deformation at a $1 \mathrm{~mm}$ gap between the parallel plates. The isothermal crystallization temperature used was $120^{\circ} \mathrm{C}$. 


\section{RESULTS AND DISCUSSION}

181

\section{(1)}

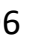

\subsection{Nonquiescent Crystallization and Rheological Properties}

Although the analysis of crystallization under quiescent conditions is important to understand the crystal structure of the material, usually the polymers processing occurs under shear flow (nonquiescent), which means the crystallization occurs under flow-induced crystallization (FIC). Consequently, the kinetics of crystallization could drastically change under this specific condition, and this aspect is particularly important technologically. The study of crystallization by rheometry allows us to verify the phenomenon of the onset time of crystallization under shear flow. As described in the literature $[10,19,20,22]$, the onset time is obtained when viscosity/shear stress as a function of time abruptly increases [10]. The increase in viscosity $(\rightarrow \infty)$ indicates that the material becomes solid, which means crystallization. This induction time is approximately proportional to the inverse of the nucleation rate $[10,20]$. 

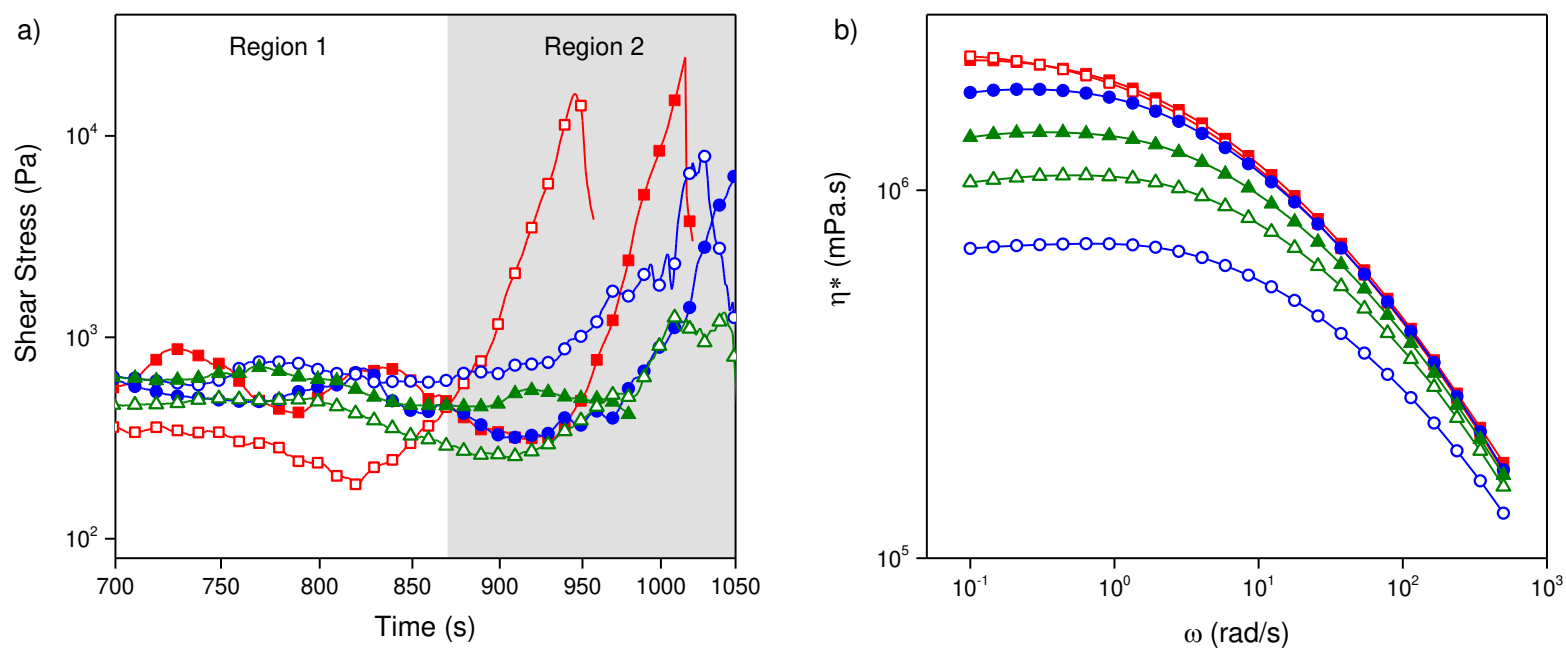

197

Figure 1 - (a) Shear rate as a function of time and (b) Complex viscosity as a function of

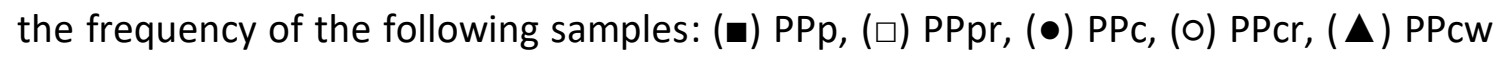
and $(\Delta)$ PPcwr.

In the FIC tests (Figure 1.a) performed at $1 \%$ strain in a linear viscoelastic range, it is possible to verify two distinct regions: Region 1 where the shear rate remains practically the same and Region 2 where the shear rate increases abruptly after some time [19]. This time is known as the beginning of crystallization and, after that, the shear rate tends to infinity [20]. It is observed that reprocessing (without the presence of contaminants) reduces the induction time, which can be attributed to a lower molar mass (Figure 1.b) and, therefore, the crystallization process occurs in less time. This behavior was also observed by Fitaroni et al. in their study on the thermal and crystallization behavior of post-consumer recycled PET [20]. It was observed that the more degraded samples showed a reduction in the time to start crystallization, which was attributed to a lower molar mass that facilitated this process. The presence of contaminants significantly alters this process, since longer induction times were observed for all contaminated 
samples. Although this is an important issue, studies on the influence of the presence of contaminants on FIC were not found in the literature.

As can be seen in Figure 1 (b), contaminated, washed, and reprocessed sample (PPcwr) showed lower complex viscosity $\left(\eta^{*}\right)$, near the unwashed sample(PPcr) and that presents the lowest value of $\eta^{*}$. Since the washed samples present lower molar mass than that shown by the contaminated one (PPC), this might mean that: (i) the washing process does not efficiently eliminate the contaminants and (ii) the washing process, combined with the reprocessing step, also contributes to the degradation of PP chains once the viscosity of PPcw is lower than that of PPc.

Paiva et al. [21] proved that the washing process, despite helping to decontaminate the material, cannot completely decontaminate the material, under the conditions carried to degradative processes in PP.

It was expected that the samples that showed the lowest molar mass, that is, PPcr and PPcwr, had a shorter induction time. As it is described in the literature $[19,20,22,24]$, the rate of crystallization is related to nucleability and transportability, so it is strongly dependent on the mobility and diffusivity of the chain. FIC is a nonequilibrium thermodynamic phase transition controlled by chain relaxation and crystallization. When the chains are subjected to a flow/shear stress, the chains are oriented and stretched. This reduces the conformational entropy, while the free energy of the polymer melts will increase by a factor of $\mathrm{T} \Delta \mathrm{S}$ that corresponds to the nucleation barrier. Therefore, the free energy associated with the nucleation barrier of the critical nucleus decreases, as described by Wang, Ma and Li [24]. On the other hand, the contaminants hinder this process once an increase in FIC time was observed despite lower molar mass. 


\section{3.2. Quiescent Crystallization} of contaminants.

The diffusion process of small molecules is mainly governed by the surrogate molar mass and temperature. During the nucleation step, they remain between the chains increasing the entropy of the system and making this process difficult. As observed by the DSC results, under quiescent conditions, the presence of contaminants also interferes with the crystallization process, reducing the $\mathrm{X}_{\mathrm{C}}$ values (Table 3 ).

The different methods applied to PPp seem to interfere with the degree of crystallinity $\left(X_{C}\right)$ of the samples (second heating). As can be seen in Table 3, there was a slight decrease in the degree of crystallinity for practically all samples compared to PPp. It is known that the crystallinity of PP can be influenced by several factors such as the molar mass, molar mass distribution, presence of additives, degradation, and processing history. In a study by Fitaroni et al., it has been reported that the presence of contaminants influences both quiescent and nonquiescent crystallization in contaminated polyethylene (PET) samples, as the contaminant acts by hindering the approximation and, consequently, the packaging of the chains [15]. Because the degree of crystallinity is related to the enthalpy of fusion, which is influenced by the diffusivity of the chains, the results indicate that the crystallinity degree decreases in the presence

The crystallization temperature upon non-isothermal cooling $\left(T_{C}\right)$ was slightly shifted to higher temperatures meaning a slight increase in crystallization kinetics. Due to the increased mobility of the shorter chains, formed as a result of degradation by chain scission (Figure 1-b), the quiescent crystallization process is slightly enhanced. Furthermore, the crystallization rate $(\Delta T)$ values, obtained by the difference between 
the crystallization onset temperature ( $T_{\text {onset }}$ ) and $T_{c}$, mainly reduce when contamination is applied (PPc).

To better understand the quiescent crystallization kinetics, the maximum time for a sample to be fully crystallized, known as $T_{\text {full, }}$ was determined. As can be seen (Table 3), although non-isothermal crystallization was slightly enhanced by higher $T_{C}$ values, the time it took for this process to complete was the same regardless of the recycling methods applied. This might be due to the presence of contaminants that slow the crystallization rate, keeping $\mathrm{T}_{\text {full }}$ unchanged.

Table 3 - DSC crystallization parameters of PP samples.

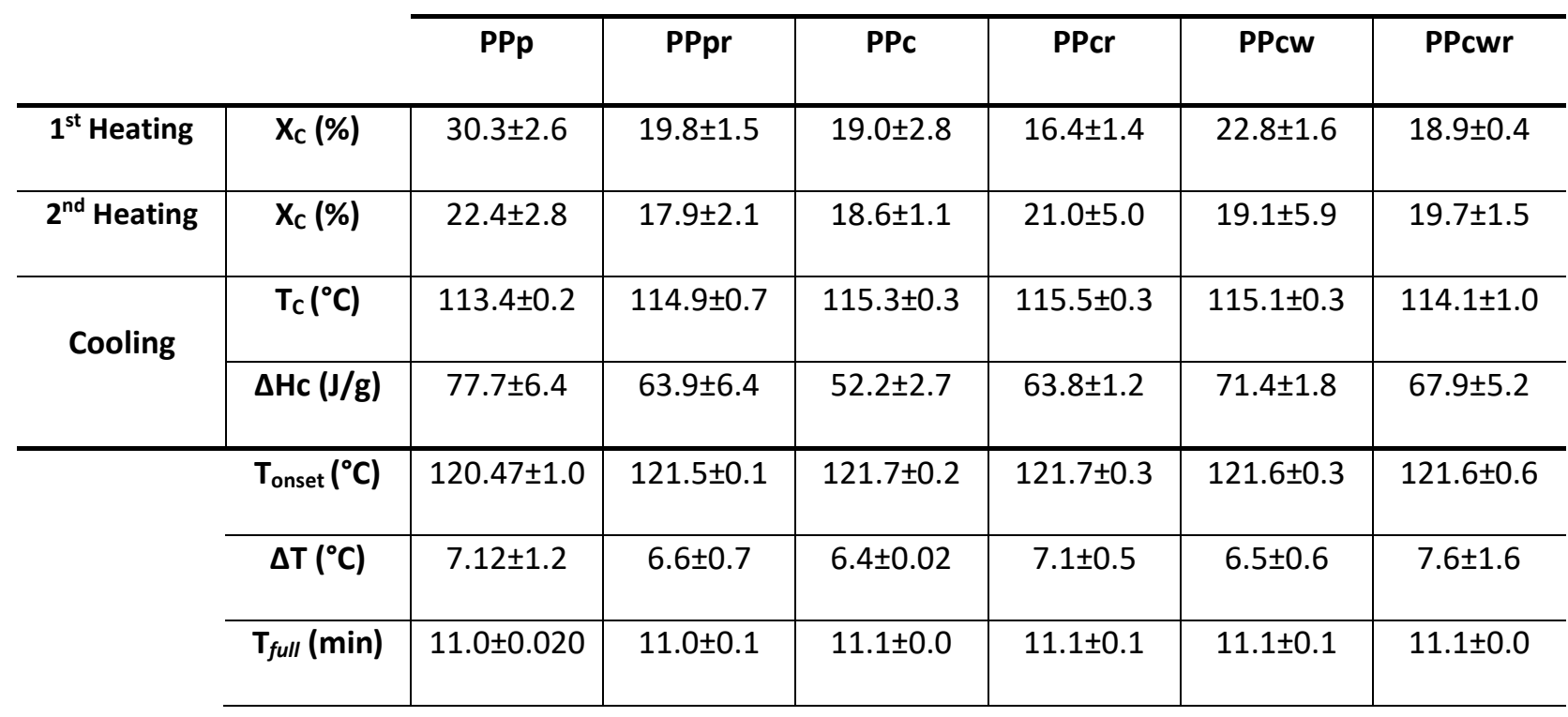

272 


\section{3.3. Thermal Analysis}

The initial mass loss $\left(T_{i}\right)$ temperature data from the thermogravimetric analysis were obtained considering the loss of $5 \%$ of the initial mass. As can be seen in Table 4, there was a decrease in the $T_{i}$ value when the material was reprocessed. PPcwr sample showed a significant reduction in Ti when compared to pristine $\mathrm{PP}$, which can be attributed to a reduction in molar mass and, therefore, the easier initial release of volatiles. On the other hand, the presence of contaminants in all samples (PPc, PPcr, PPcw, PPcwr) increased the maximum speed mass loss temperature ( $\left.T_{\max }\right)$ determined by the maximum value of the first derivative, even with a reduction in molar mass. This behavior is reflected in another parameter, $\Delta T$, which represents the difference between $T_{\max }$ and $T_{i}$ and allows to obtain information on the mass loss kinetics.

The presence of contaminants significantly affects the kinetics of mass loss. Clearly, an increase in $\Delta T$ can be observed, indicating that the presence of contaminants alters the internal structure of the polymer in a way that hinders the production of volatiles. Fitaroni et al. [14] observed similar behavior on the influence of montmorillonite clay on the thermal stability of PP. $T_{i}$ values were not significantly changed, but an increase in $T_{\max }$ was observed with increasing clay content. This effect was attributed to an increase in the mean free path for oxygen entry and volatile exit due to the presence of clay, meaning slower gas diffusion inside the polymer matrix. 
Table 4 - Parameters obtained from TGA curves for PP samples.

303

304

a)

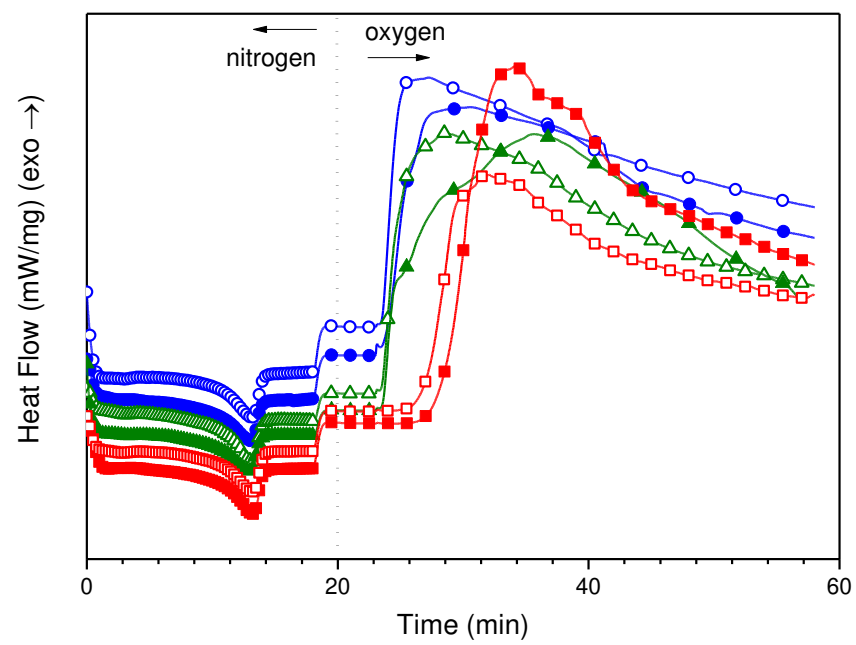

b)

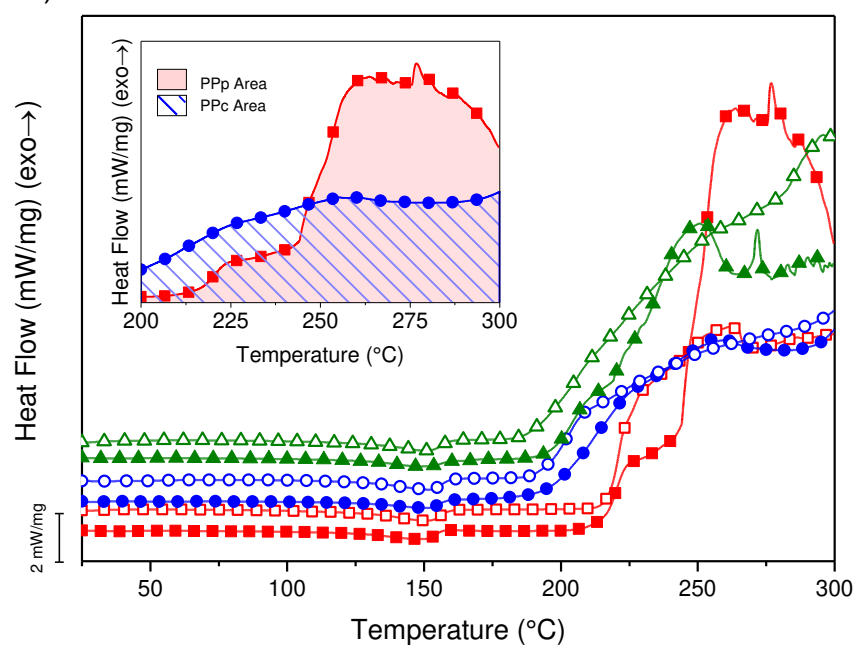

308

$\multimap \mathrm{PPp} \multimap-\mathrm{PPpr} \rightarrow \mathrm{PPc} \multimap \mathrm{PPcr} \leadsto \mathrm{PPcw} \backsim-\mathrm{PPcwr}$

Figure 2 - OIT curves for PP samples (a) and (b) OITD curves for PP samples.

When comparing the reprocessed sample with the pristine PP, a reduction in the

dynamic and conventional OIT is observed, which indicates that it is more susceptible to samples showed an even more significant reduction in induction time. This indicates that 
contaminants catalyze the degradation reaction of contaminated polypropylene $[14,17]$.

A previous study [6], carried out on the same set of samples, indicated that both the washing process and the reprocessing help in decontaminating the material. However, as previously described, several residual contaminants were still observed, which was confirmed by their migration through direct contact with food simulants, where the extractability tests were carried out using solid-phase microextraction (SPME) and analyzed by gas chromatography coupled to the mass spectrometer (GC-MS). As shown by the rheometry results, the applied washing process was not enough to eliminate all reaction (Tre* for PP samples.

330

Table 5 - Average thermal analysis parameters for PP samples.

\begin{tabular}{|c|c|c|c|c|c|c|c|}
\hline & Parameter & PPp & PPpr & PPc & PPcr & PPcw & PPcwr \\
\hline OIT & Time (min) & $6.3 \pm 0.8$ & $4.9 \pm 1.1$ & $0.9 \pm 0.1$ & $0.6 \pm 0.0$ & $0.7 \pm 0.1$ & $0.4 \pm 0.2$ \\
\hline OITD & $\mathrm{T}_{\text {exo }} *\left({ }^{\circ} \mathrm{C}\right)$ & $214.5 \pm 5.6$ & $216.8 \pm 4.6$ & $192.2 \pm 0.8$ & $189.4 \pm 0.1$ & $193.1 \pm 0.8$ & $189.8 \pm 4.9$ \\
\hline TGA & $\mathrm{Ti}\left({ }^{\circ} \mathrm{C}\right)$ & $229.6 \pm 5.5$ & $232.6 \pm 1.3$ & $229.8 \pm 1.5$ & $232.5 \pm 1.3$ & $226.7 \pm 0.9$ & $218.5 \pm 7.2$ \\
\hline & $\mathrm{Ti}-\mathrm{T}_{\text {exo }} *\left({ }^{\circ} \mathrm{C}\right)$ & $15.0 \pm 11.1$ & $15.8 \pm 3.4$ & $37.6 \pm 0.8$ & $43.1 \pm 1.2$ & $33.5 \pm 0.2$ & $28.7 \pm 2.4$ \\
\hline
\end{tabular}


Oxidation induction temperature (OITD) tests are an important tool in the evaluation of the thermal stability of polymers, especially those that undergo thermo-oxidation, such as PP. The technique is beneficial once the diffusion of oxygen through the sample occurs during all the analysis, in the same conditions as TGA. As the heat flow is measured, it is possible to identify when the exothermal oxidation started and associate it with the mass loss obtained by TGA. It is observed that the samples that went through the contamination process showed a decrease in the temperature at the beginning of the exothermic reactions. These data are contradictory and can be the basis for questioning the use of thermogravimetric analysis as the only technique to determineing thermal stability, which has already been observed by Fitaroni et al. [14] for multicomponent polymeric materials using an inorganic filler. To the best of our knowledge, for pure polymers and contaminated ones, there is no discussion about this limitation, which must be considered in all evaluations using TGA. The oxidative degradation reaction is one of the most important mechanisms for PP degradation[14,17]. Also, as described previously, the presence of contaminants significantly influences this parameter, as may be observed when $T_{\text {exo }}$ is compared with the sample without (PPp and PPpr) and with contaminants (PPc, PPcr, PPcw and PPcwr). For all samples that present contaminants, the initial time of the exothermic reactions does not seem to change much. However, additional information could be evaluated by analyzing the different heat profiles for each sample. For the more degraded samples, there is less variation in the heat flow. On the other hand, the heat flow variation, which can be analyzed by the area under the curve of the heat flow variation as a function of temperature for the least degraded samples (PPp) is significantly higher, indicating that more samples can be degraded, as shown in Figure 2 (b). 


\section{CONCLUSION}

The effect of the presence of contaminants in recycled polypropylene on the quiescent and nonquiescent crystallization was evaluated. Their effect on the thermal stability of the samples was more accurate when TGA analysis and OIT (conventional and dynamic) were used as a complementary technique. The results indicate that the residual contaminants decrease the molar mass, although a reduction in flow-induced crystallization time is expected. However, the contaminants' induction times were increased for all contaminated samples. On the other hand, in quiescent conditions, the maximum time for a sample to be fully crystallized ( $\left.T_{\text {full }}\right)$ remains constant. This behavior conditions.

\section{ACKNOWLEDGMENTS}

The authors would like to acknowledge FAPESP $2017 / 24316-8$ and 2016/25703-2. 


\section{Conflict of Interest}

The authors confirm that they have no conflicts of interest with respect to the work described in this manuscript.

\section{Availability of data and material}

The data are in public repository at UFSCar: https://repositorio.ufscar.br/

\section{REFERENCES}

[1] D.M. Panaitescu, Z. Vuluga, C. Radovici, C. Nicolae, Morphological investigation of PP/nanosilica composites containing SEBS, Polym. Test. 31 (2012) 355-365. https://doi.org/10.1016/j.polymertesting.2011.12.010.

[2] N. Parashar, S. Hait, Science of the Total Environment Plastics in the time of COVID-19 pandemic : Protector or polluter?, Sci. Total Environ. 759 (2021) 144274. https://doi.org/10.1016/j.scitotenv.2020.144274.

[3] A.L. Patrício Silva, J.C. Prata, T.R. Walker, A.C. Duarte, W. Ouyang, D. Barcelò, T. Rocha-Santos, Increased plastic pollution due to COVID-19 pandemic: Challenges and recommendations, Chem. Eng. J. 405 (2021) 126683. https://doi.org/10.1016/j.cej.2020.126683.

[4] US Food and Drug Administration (FDA), Guidance for industry: Use of recycled plastics in food packaging, Chem. Considerations. HFS-275. Washington, DC US FDA, Cent. Food Saf. Appl. Nutr. 4 (2006).

[5] ANVISA, Resolução-DOU $n^{\circ}-228$, de 30 de novembro de 2010. Regulamento técnico MERCOSUL sobre migração em materiais, embalagens e equipamentos 
plásticos destinados a entrar em contato com alimentos., Diário Of. Da União, Brasília, DF. 1 (2010) 105. https://doi.org/10.1017/CBO9781107415324.004.

[6] R. Paiva, M. Wrona, C. Nerín, I. Bertochi Veroneze, G.-L. Gavril, S. Andrea Cruz, Importance of profile of volatile and off-odors compounds from different recycled polypropylene used for food applications, Food Chem. (2021) 129250. https://doi.org/10.1016/j.foodchem.2021.129250.

[7] P.S. Garcia, S.A. Cruz, C. Nerín, Comparison of different extrusion processes for cleaning the recycled polypropylene removing volatile and non-volatile contaminants, Prog. Rubber, Plast. Recycl. Technol. 30 (2014) 37-54. https://doi.org/10.1177/147776061403000103.

[8] S.A. Cruz, É.C. Oliveira, F.C.S. De Oliveira, P.S. Garcia, M.L.Q.A. Kaneko, Polímeros reciclados para contato com alimentos, Polimeros. 21 (2011) 340345. https://doi.org/10.1590/S0104-14282011005000052.

[9] P.S. Garcia, C.H. Scuracchio, S.A. Cruz, Effect of residual contaminants and of different types of extrusion processes on the rheological properties of the postconsumer polypropylene, Polym. Test. 32 (2013) 1237-1243. https://doi.org/10.1016/j.polymertesting.2013.08.002.

[10] L.B. Fitaroni, É.C. de Oliveira, A.L. Marcomini, C.M. Paranhos, F.L. Freitas, S.A. Cruz, Reprocessing and Solid State Polymerization on Contaminated Postconsumer PET: Thermal and Crystallization Behavior, J. Polym. Environ. 28 (2020) 91-99. https://doi.org/10.1007/s10924-019-01579-9.

[11] M.A.B. de M. S. A. Cruz, M. Zanin, P. A. P. Nascente, Superficial Modification in Recycled PET by Plasma Etching for Food Packaging, J. Appl. Polym. Sci. Appl. 
Polym. 115 (2010) 2728-2733. https://doi.org/10.1002/app.29958.

[12] S.A. Cruz, M. Zanin, Evaluation and identification of degradative processes in post-consumer recycled high-density polyethylene, Polym. Degrad. Stab. 80 (2003) 31-37. https://doi.org/10.1016/S0141-3910(02)00379-8.

[13] S.A. Cruz, C.H. Scuracchio, L.B. Fitaroni, C. Oliveira, The use of melt rheology and solution viscometry for degradation study of post-consumer poly(ethylene terephthalate): The effects of the contaminants, reprocessing and solid state polymerization, Polym. Test. 60 (2017) 236-241. https://doi.org/10.1016/j.polymertesting.2017.03.026.

[14] L.B. Fitaroni, J.A. De Lima, S.A. Cruz, W.R. Waldman, Thermal stability of polypropylene-montmorillonite clay nanocomposites: Limitation of the thermogravimetric analysis, Polym. Degrad. Stab. 111 (2015) 102-108. https://doi.org/10.1016/j.polymdegradstab.2014.10.016.

[15] S. Palkopoulou, C. Joly, A. Feigenbaum, C.D. Papaspyrides, P. Dole, Critical review on challenge tests to demonstrate decontamination of polyolefins intended for food contact applications, Trends Food Sci. Technol. 49 (2016) 110120. https://doi.org/10.1016/j.tifs.2015.12.003.

[16] C. Science, Mechanical Reprocessing of Polyolefin Waste : A Review, (2015). https://doi.org/10.1002/pen.

[17] T. Mekonnen, Thermo-mechanical degradation of polypropylene (PP) and lowdensity polyethylene (LDPE) blends exposed to simulated recycling, Polym. Degrad. Stab. (2020) 109390. https://doi.org/10.1016/j.polymdegradstab.2020.109390. 
[18] C. Spicker, N. Rudolph, I. Kühnert, C. Aumnate, The use of rheological behavior to monitor the processing and service life properties of recycled polypropylene, Food Packag. Shelf Life. 19 (2019) 174-183.

https://doi.org/10.1016/j.fpsl.2019.01.002.

[19] J. Van Meerveld, Towards a rheological classification of flow induced crystallization experiments of polymer melts, (2004) 119-134. https://doi.org/10.1007/s00397-004-0382-7.

[20] S. Coppola, N. Grizzuti, Microrheological Modeling of Flow-Induced Crystallization, Macromolecules (2001) 5030-5036. https://doi.org/10.1021/ma010275e

[21] R. Paiva, I. Bertochi, V. Magdalena, W. Cristina, N. Sandra, A. Cruz, The Role of Residual Contaminants and Recycling Steps on Rheological Properties of Recycled Polypropylene, J. Polym. Environ. (2021). https://doi.org/10.1007/s10924-021-02214-2.

[22] S.A. Cruz, L.A. Onoue, C.M. Paranhos, E. Longo, Effect of sepiolite on the quiescent and non-quiescent crystallization behaviour of the biodegradable poly(Lactic acid) prepared via casting and melting, Express Polym. Lett. 13 (2019) 825-834. https://doi.org/10.3144/expresspolymlett.2019.71.

[23] R. Lieberman, C. Stewart, Propylene Polymers, Encycl. Polym. Sci. Technol. 11 (2004). https://doi.org/10.1002/0471440264.pst301.

[24] Z. Wang, Z. Ma, L. Li, Flow-Induced Crystallization of Polymers : Molecular and Thermodynamic Considerations, (2016). https://doi.org/10.1021/acs.macromol.5b02688. 
473 [1] D.M. Panaitescu, Z. Vuluga, C. Radovici, C. Nicolae, Morphological investigation of PP/nanosilica composites containing SEBS, Polym. Test. 31 (2012) 355-365. https://doi.org/10.1016/j.polymertesting.2011.12.010.

[2] N. Parashar, S. Hait, Science of the Total Environment Plastics in the time of

[5] ANVISA, Resolução-DOU n-228, de 30 de novembro de 2010. Regulamento técnico MERCOSUL sobre migração em materiais, embalagens e equipamentos plásticos destinados a entrar em contato com alimentos., Diário Of. Da União, Brasília, DF. 1 (2010) 105. https://doi.org/10.1017/CBO9781107415324.004.

[6] R. Paiva, M. Wrona, C. Nerín, I. Bertochi Veroneze, G.-L. Gavril, S. Andrea Cruz, Importance of profile of volatile and off-odors compounds from different recycled polypropylene used for food applications, Food Chem. (2021) 129250. https://doi.org/10.1016/j.foodchem.2021.129250. 
contaminants, Prog. Rubber, Plast. Recycl. Technol. 30 (2014) 37-54. https://doi.org/10.1177/147776061403000103.

[8] S.A. Cruz, É.C. Oliveira, F.C.S. De Oliveira, P.S. Garcia, M.L.Q.A. Kaneko, Polímeros reciclados para contato com alimentos, Polimeros. 21 (2011) 340345. https://doi.org/10.1590/S0104-14282011005000052.

[9] P.S. Garcia, C.H. Scuracchio, S.A. Cruz, Effect of residual contaminants and of different types of extrusion processes on the rheological properties of the postconsumer polypropylene, Polym. Test. 32 (2013) 1237-1243. https://doi.org/10.1016/j.polymertesting.2013.08.002.

[10] L.B. Fitaroni, É.C. de Oliveira, A.L. Marcomini, C.M. Paranhos, F.L. Freitas, S.A. Cruz, Reprocessing and Solid State Polymerization on Contaminated Postconsumer PET: Thermal and Crystallization Behavior, J. Polym. Environ. 28 (2020) 91-99. https://doi.org/10.1007/s10924-019-01579-9.

[11] M.A.B. de M. S. A. Cruz, M. Zanin, P. A. P. Nascente, Superficial Modification in Recycled PET by Plasma Etching for Food Packaging, J. Appl. Polym. Sci. Appl. Polym. 115 (2010) 2728-2733. https://doi.org/10.1002/app.29958.

[12] S.A. Cruz, M. Zanin, Evaluation and identification of degradative processes in post-consumer recycled high-density polyethylene, Polym. Degrad. Stab. 80 (2003) 31-37. https://doi.org/10.1016/S0141-3910(02)00379-8.

[13] S.A. Cruz, C.H. Scuracchio, L.B. Fitaroni, C. Oliveira, The use of melt rheology and solution viscometry for degradation study of post-consumer poly(ethylene terephthalate): The effects of the contaminants, reprocessing and solid state polymerization, Polym. Test. 60 (2017) 236-241. 
https://doi.org/10.1016/j.polymertesting.2017.03.026.

[14] L.B. Fitaroni, J.A. De Lima, S.A. Cruz, W.R. Waldman, Thermal stability of polypropylene-montmorillonite clay nanocomposites: Limitation of the thermogravimetric analysis, Polym. Degrad. Stab. 111 (2015) 102-108. https://doi.org/10.1016/j.polymdegradstab.2014.10.016.

[15] S. Palkopoulou, C. Joly, A. Feigenbaum, C.D. Papaspyrides, P. Dole, Critical review on challenge tests to demonstrate decontamination of polyolefins intended for food contact applications, Trends Food Sci. Technol. 49 (2016) 110120. https://doi.org/10.1016/j.tifs.2015.12.003.

[16] C. Science, Mechanical Reprocessing of Polyolefin Waste : A Review, (2015). https://doi.org/10.1002/pen.

[17] T. Mekonnen, Thermo-mechanical degradation of polypropylene (PP) and lowdensity polyethylene (LDPE) blends exposed to simulated recycling, Polym. Degrad. Stab. (2020) 109390. https://doi.org/10.1016/j.polymdegradstab.2020.109390.

[18] C. Spicker, N. Rudolph, I. Kühnert, C. Aumnate, The use of rheological behavior to monitor the processing and service life properties of recycled polypropylene, Food Packag. Shelf Life. 19 (2019) 174-183. https://doi.org/10.1016/j.fpsl.2019.01.002.

[19] J. Van Meerveld, Towards a rheological classification of flow induced crystallization experiments of polymer melts, (2004) 119-134. https://doi.org/10.1007/s00397-004-0382-7.

[20] S. Coppola, N. Grizzuti, Microrheological Modeling of Flow-Induced 
Crystallization, (2001) 5030-5036.

[21] R. Paiva, I. Bertochi, V. Magdalena, W. Cristina, N. Sandra, A. Cruz, The Role of Residual Contaminants and Recycling Steps on Rheological Properties of Recycled Polypropylene, J. Polym. Environ. (2021). https://doi.org/10.1007/s10924-021-02214-2.

[22] S.A. Cruz, L.A. Onoue, C.M. Paranhos, E. Longo, Effect of sepiolite on the quiescent and non-quiescent crystallization behaviour of the biodegradable poly(Lactic acid) prepared via casting and melting, Express Polym. Lett. 13 (2019) 825-834. https://doi.org/10.3144/expresspolymlett.2019.71.

[23] R. Lieberman, C. Stewart, Propylene Polymers, Encycl. Polym. Sci. Technol. 11 (2004). https://doi.org/10.1002/0471440264.pst301.

[24] Z. Wang, Z. Ma, L. Li, Flow-Induced Crystallization of Polymers : Molecular and Thermodynamic Considerations, (2016). https://doi.org/10.1021/acs.macromol.5b02688. 


\section{Supplementary Files}

This is a list of supplementary files associated with this preprint. Click to download.

- renamed0a3d6.pdf 